\title{
Systematic Literature Review Protocol for Software Outsourcing Partnership (SOP)
}

\author{
Sikandar Ali ${ }^{1,2}$, Siffat Ullah Khan ${ }^{1,2,3}$ \\ ${ }^{1,}$ Software Engineering Research Group SERG_UOM, \\ ${ }^{1}$ Department of Computer Science \& IT, \\ ${ }^{3}$ Department of Software Engineering, \\ University of Malakand, Khyber Pakhtunkhwa,Pakistan
}

\begin{abstract}
:
CONTXEXT:-Software outsourcing partnership (SOP) is a relationship between client and vendor organizations for shared goals. A SOP is different than ordinary outsourcing contractual relationship. Usually a successful outsourcing relationship may lead to outsourcing partnership.

OBJECTIVE:-The objective of this research is to identify factors via systematic literature review (SLR), that are significant to be developed by outsourcing vendor organization which lead them to convert existing outsourcing contractual relationship into outsourcing partnership with client organization.

METHOD: -SLR will be used for the aforementioned objective. SLR is based on a structured protocol and is more thorough than ordinary review.

EXPECTED OUTCOMES: - We have developed a SLR protocol for the SOP. The anticipated outcome of this review will be a list of critical success factors (CSFs) and critical risks (CRs) which can have a positive or a negative role in building or converting the existing outsourcing relationship into outsourcing partnership.
\end{abstract}

Keywords: Client-Vendor Relationship, Software Outsourcing partnership, Systematic Literature Review Protocol

\section{INTRODUCTION}

In recent year, software outsourcing organizations have paid much attention to build a successful partnership between the client and vendor organizations $[1,2]$.

Several firms have established close relationships with service providers (SPs) like UPS and Motorola organisations [3], Kodak, IBM and DEC [4],SDB and Hi Sun [5], USAA and IBM [6], and Xerox and EDS [7]. Organization usually establishes partnership with counterpart organization after identifying the limitation of legal contracts. Till 1990s, many software outsourcing organizations experienced difficulties in establishing and managing a successful outsourcing relationship with SPs because contractual nature of outsourcing is shifting to partnership [1].

\subsection{What is software outsourcing?}

Software development outsourcing is a contract-based relationship between clients and vendors organizations in which client(s) contract out all or part of its software development activities to a vendor(s), who provide agreed services in return for remuneration from client organization [8]. Software outsourcing is defined as the process of turning over all or part of (a client) organization software development activities to an external SPs [8, 9]. Software outsourcing is practiced from the last two decades and client in the developed countries are outsourcing their projects to the developing countries to get high quality software at low cost.

\subsection{What is software outsourcing partnership?}

Partnership can be defined as "a tailored business relationship based upon mutual trust, openness, shared risk, and shared rewards that yield a competitive advantage, resulting in business performance greater than would be achieved by the firms individually" [10].

Software outsourcing partnership can be defined as "an inter-organizational software development relationship between vendor and client organizations to achieve the participants shared goals" [11]

According to Kishore et al [12] they Suggest a framework of four outsourcing relationship types (FORT). These are support, alignment, reliance, and alliance. A relationship in which ownership substitution and strategic impact is high, the relationship is characterized as an alliance. In an alliance relationship the degree of trust is considered to be high and the contractual control low. Outsourcing partnership is the form of an alliance relationship [2,13,14].

Software outsourcing partnership is considered more beneficial as compared to contractual outsourcing relationship as contractual relationship bears a number of barriers, e.g. (1) difficulties in writing a complete 
contract (2) Investment from one or both parties on relation specific assets (3) Term of contract is long (4) Amount of contract is high (5) Many clients implement total IT outsourcing [22, 23] (6) Insufficient Management of the risk associated with complex software projects in Contract [24] . (7) The uncertainty of IT outsourcing are increasing because of unrealistic estimation of cost, manpower requirements and time [24]. (8) It will be hard to apply the results because the outcome often don't match the actual results [24].

Partnership Relationship provides the mechanism for (1) Protecting relations specific assets investments and promote further investments.(2) sustaining long-term relationships.(3) dealing with uncertainty and (4) reducing risk [22, 23].

\subsection{Outsourcing relationship}

\section{BACKGROUND}

Theoretically, software development outsourcing can be viewed in literature from three different perspectives, strategic management, economic and social perspectives [1]. Strategic management perspective is based on resource and resource dependency theories, it explains how firms implement outsourcing paradigm to achieve desired goals [1]. However it does not consider relationship management aspect [1]. Economic perspective is based on transaction cost and agency theories. It examines coordination, efficiency and governance of economic transactions between firms [15]. But it does not consider factors that affect organizations having other reasons to outsource beside cost efficiency [1]. On the other hand social perspective is based on social exchange and relational exchange theories, it focuses on relationship between the service receivers and the service providers [1]. It is differentiated from the other perspectives by the assumption that there are shared norms and an agreement of interests between the parties and leading to considerations of the issues like trust, equity, and cooperation [16]. In social perspective the formal contract is necessary but not sufficient for the success of outsourcing [17]. It implies that the termination or continuation of client-vendor relationships is based on the satisfaction of the both parties [15].

A number of studies have emphasized on the importance of relationships in software development outsourcing. The relationships between client(s) and vendor(s) organizations plays a vital role in the success or failure of the outsourcing arrangement [16]. A key to achieve benefits from IT outsourcing is maintaining positive client-vendor relationships [18]. Building and sustaining a flexible relationship between clients and vendors of IT outsourcing services is one of the ways to create values in outsourcing [19]. Understanding the relationship that arises in IT outsourcing is critical, since it comes about not only through the operationalization of the contract but also as a natural consequence of the resulting issues of dependency [20]. Along with the increasing practice of IS/IT outsourcing the nature of the outsourcing is shifting from a pure contractual to the one based on relationship [1]. Many organizations experienced difficulties in forming and managing a successful outsourcing relationship with SPs as the nature of outsourcing evolved from a contract relationship between the service receiver and provider to a partnership relationship [21].

\subsection{Existing literature on software outsourcing partnership}

Previous research (e.g. [25]) classified the relationship between organizations into two types: transactional style relationship (develops through a formal contract in which the rules are well specified and the failure to deliver on commitments by either party is resolved through either litigation or penalty clauses in the contract). and partnership style relationship (involves risk and benefit sharing, the need to view the relationship as a series of exchanges without a definite endpoint, and the need to establish a range of mechanisms to monitor and execute its operations) [26].

In terms of partnership there are two types of outsourcing 1) Asset Outsourcing: it involves transfer of assets such as hardware, software, and people to service providers. 2) Service outsourcing: it involves system integration and system management services without asset transfer [11].

The success of IT outsourcing partnership needs the six-levels integration to achieve sufficient communication, cooperation and control. These six levels integration include: strategic integration, tactic integration, operational integration, interpersonal integration, cultural integration and knowledge integration [22].

There are two types of criteria for the success of outsourcing partnership (1) Measurable criteria which include realization of goals, realization of service levels, expansion of the scope of the contract, renewal of the contract and (2) soft criteria which includes customer satisfaction, active communication, involvement, cultural fit and trust. [27].

\section{RESEARCH METHOD}

A detailed methodological approach is necessary in any kind of literature review. Three general kinds of literature reviews have been reported in the literature [28] which are as follows. 
A. Theoretical background: This is a section/portion of a journal article that gives the context and theoretical foundations of the research, and is most commonly labeled as theoretical background or literature review [28].

B. Thesis literature review: This is chapter of a graduate thesis and is labeled as thesis literature review [28].

C. Stand-alone literature review: This is a journal-length article whose main purpose is to review the literature in a field, without any primary data. When a stand-alone literature review is conducted using a systematic, rigorous standard, it is called a systematic literature review (SLR) or simply Systematic review [28]. This method has been widely implemented in some disciplines such as medicine, software engineering, computer graphics etc. Since 2004 systematic review has become an important methodology of evidence-bases software engineering (EBSE), and many SLRs have been conducted and reported [29].

\subsection{Systematic Literature Review (SLR) Process}

Systematic literature review has three main phases as described by Kitchenham [29]. These are planning the review, conducting the review, and reporting the review. In this paper we describe the SLR protocol which is the first phase of a systematic literature review (i.e. planning the review).

\subsection{SLR Protocol Development}

Prior to conduct the systematic review, we developed a review protocol. A pre-defined protocol reduces researcher bias and increases the rigor and repeatability of the review [29]. An SLR protocol specifies the review plan and procedures by describing the details of various strategies for performing the systematic review. In particular, it defines background context for the research, the research questions, search strategy to identify the relevant literature, inclusion and exclusion criteria for selecting relevant studies, the treatment of publication quality assessment, the data extraction plan, the data synthesis plan and the methodology for extracting and synthesizing information in order to address the research questions [29]. Fig.1 depicts the various stages in the SLR process.

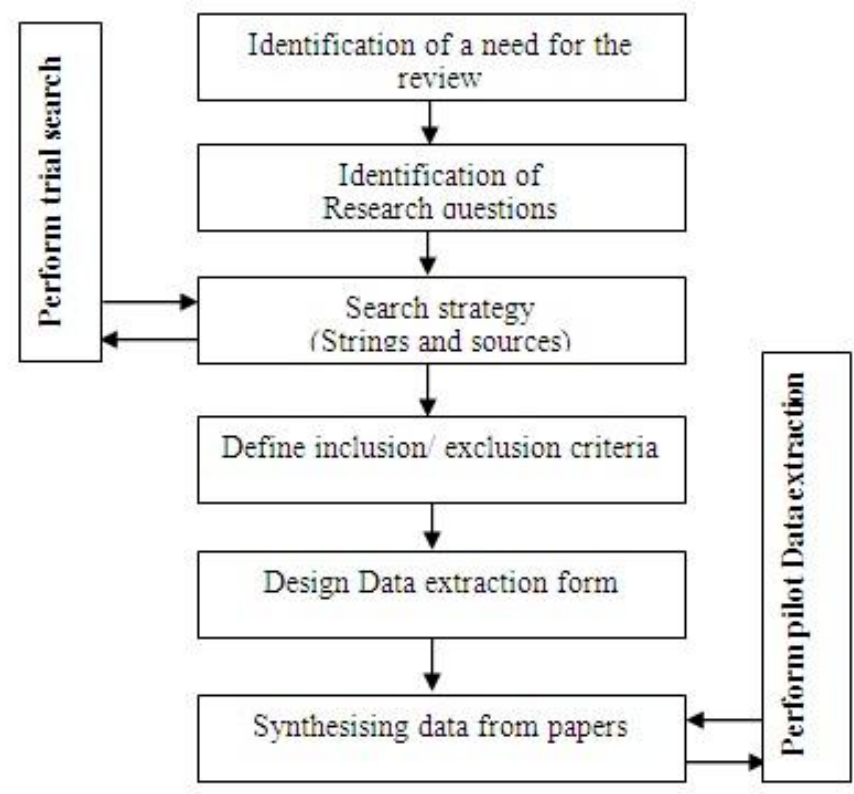

Fig 1.Development process for the Systematic Literature Review Protocol

\subsection{Research Question}

However, despite the importance of partnership outsourcing, no systematic literature review has been carried out on partnership outsourcing practice in general and the identification of factors that have a significant impact on vendor organizations in particular. To do this we intend to address the following research question.

RQ1. What are the critical success factors, as identified in the literature, to be developed by software outsourcing vendor organizations which assist in promoting the exiting outsourcing relationship into partnership with client organization.

RQ2. What are the critical risks, as identified in the literature, involved from software vendors perspective in promoting the exiting outsourcing relationship into partnership with client organization.

\subsection{Construction of Search Terms}

Defining the elements for the research questions as shown below will help in designing relevant and accurate search term(s)/string(s). 
- $\quad$ Population: Vendors and clients involved in outsourcing partnership.

- Intervention: Factors, Characteristics, Risks, partnership builders, Relationship promoters.

- Outcomes of relevance: Establishing software outsourcing Partnership, Partnership maintenance, Promoting existing outsourcing relationship into partnership, capabilities of outsourcing vendors in existing project

- $\quad$ Experimental Design: Empirical studies, systematic review, theoretical studies, case studies, experts' opinions

An example of the research question containing the above details is:

\section{RQ1: What factors/characteristics]"INTERVENTION"}

Developed by

[Vendors and clients involved in Outsourcing] "POPULATION"

Which assist to

[Promote the existing outsourcing relationships to partnership] "OUTCOMES OF RELEVANCE"

\section{SEARCH STRATEGY}

We describe our search strategy from the following dimensions

- Search scope ( time and space)

- Search method i.e. automatic search or manual search (By Automatic search we mean the search performed by executing search strings on search engines of electronic data sources, whereas by manual search we mean search performed by browsing through specific journals or conference proceedings).

- Electronic data sources used. Electronic data sources mean both index engines (e.g., web of science and EI Compendex) and publishers' sites (e.g., ScienceDirect and IEEEXplore).

- Search strings

- Search validation

- Search Documentation

- Search result Management

4.1 Search scope (time and space)

We will Search for all published literature related to our research questions without any time (years) boundary/limit.

\subsection{Search method}

We will use automated search on publishers' sites only. The following publishers' sites will be searched.

- IEEE Xplore(www.ieeexplore.ieee.org)

- ACM Portal (www.acm.org)

- $\quad$ Science Direct (www.sciencedirect.com)

- CiteSeer Digital Library (citeseer.ist.psu.edu)

- Google Scholar (scholar.google.com)

- $\quad$ SpringerLink (www.springerlink.com)

\subsection{Search strings}

We have divided the search string in three types of sub search string.

A. Trial search string: Trial search string mean initial search string used in trial search

B. Lengthy search string: Lengthy search string mean search string obtained after combining major terms and its synonyms using Boolean operators. We will use this search string as our search term if database allow.

C. Smaller sub search string: By smaller sub string we mean search string obtained after search term breakup. As some of the databases do not allow lengthy search strings we will split the search term into smaller sub strings and will do separate search for each of these search strings. Finally we will summarize the search results by removing the redundancy.

\subsubsection{Trial Search}

We conducted a trial search on ScienceDirect digital library using search string given below. The results will be used for validation of major search terms.

(Partnership OR "Outsourcing partnership") AND ("Software outsourcing” OR "IT outsourcing" OR "IS outsourcing"). 


\subsubsection{Identifying search terms}

The following steps (search strategy) are used for the construction of search terms

Step1: Derivation of major terms

Use the research questions for the derivation of major terms, by identifying population, intervention and outcome.

\section{Step2: Identification of alternative spellings and synonyms}

Find the alternative spellings and synonyms; for these major terms;

Step3 verification of key words

Verify the key words in any relevant paper;

\section{Step4 Use of Boolean Operators for conjunction}

Use Boolean Operators for conjunction if the database allows, in such a way, to use 'OR' operator for the concatenation of alternative spellings and synonyms whereas 'AND' for the concatenation of major terms. The above four steps are shown in Fig.2.

Use the research questions for the derivation of maior terms

Software Outsourcing, partnership, vendor, factors, promote, client, outsourcing relationship, relationship promotion process, risks

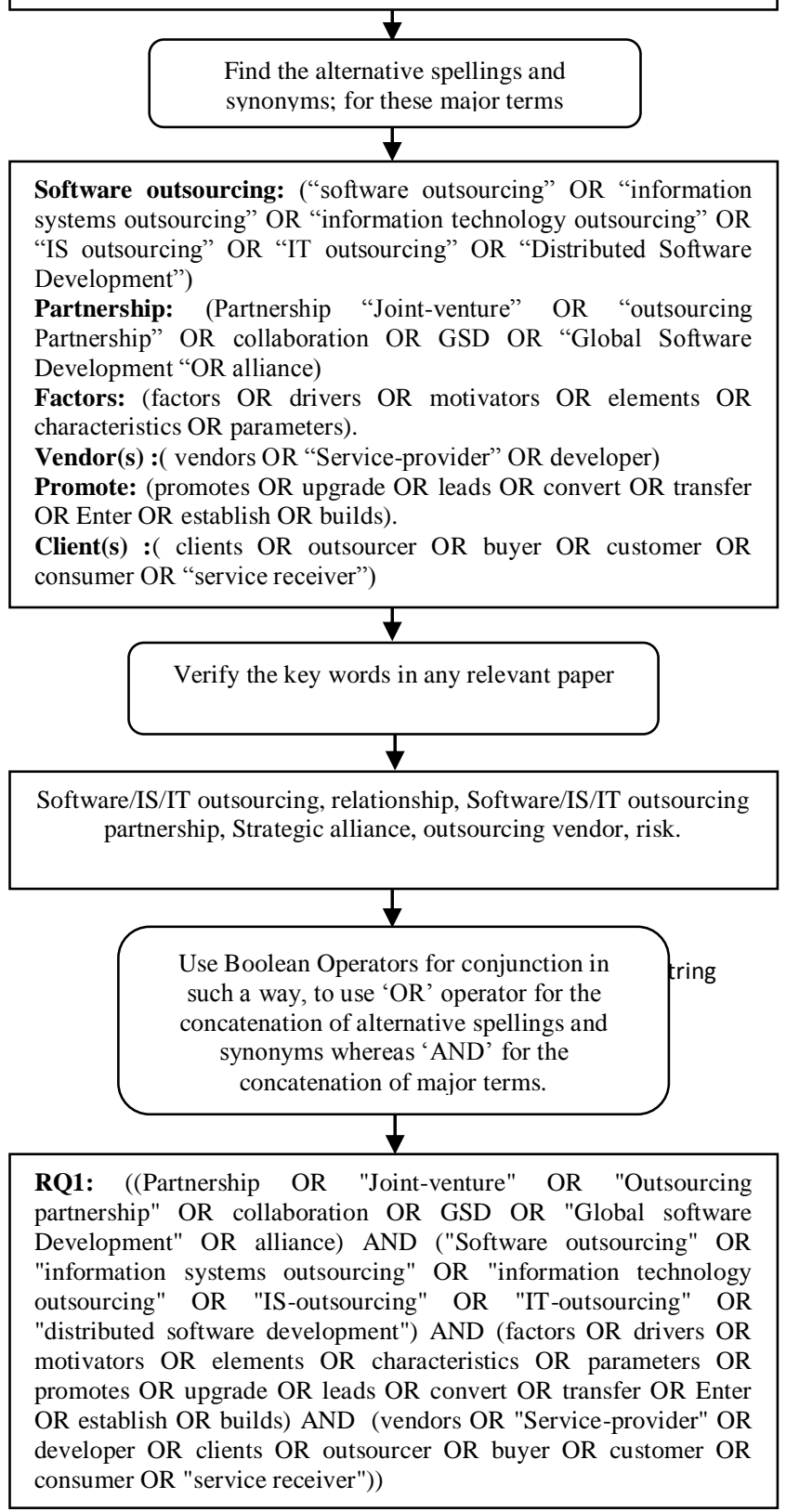


RQ1: ((Partnership OR "Joint-venture" OR "Outsourcing partnership" OR collaboration OR GSD OR "Global software Development" OR alliance) AND ("Software outsourcing" OR "information systems outsourcing" OR "information technology outsourcing" OR "IS-outsourcing" OR "IT-outsourcing" OR "distributed software development") AND (factors OR drivers OR motivators OR elements OR characteristics OR parameters OR promotes OR upgrade OR leads OR convert OR transfer OR Enter OR establish OR builds) AND (vendors OR "Service-provider" OR developer OR clients OR outsourcer OR buyer OR customer OR consumer OR "service receiver"))

RQ2: ((Partnership OR "Joint-venture" OR "Outsourcing partnership" OR collaboration OR GSD OR "Global software Development" OR alliance) AND ("Software outsourcing" OR "information systems outsourcing" OR "information technology outsourcing" OR "IS-outsourcing" OR "IT-outsourcing" OR "distributed software development") AND (risks OR barriers OR challenges OR "Negative impacts" OR obstacles OR hurdles OR promotes OR upgrade OR leads OR convert OR transfer OR Enter OR establish OR builds) AND (vendors OR "Service-provider" OR developer OR clients OR outsourcer OR buyer OR customer OR consumer OR "service receiver"))

\subsubsection{Search Term Break up}

We will first use the search strings RQ1 and RQ2 mentioned as our search terms. As some of the databases (i.e Google Scholar and Springer Link) don't allow the lengthy search strings we will split the search term into smaller sub strings and will do separate search for each of these search strings. Finally we will summarize the search results by removing the redundancy.

The breakup of the RQ1 and RQ2 are mentioned in the form smaller strings are given below.

\subsubsection{Smaller sub search string}

String1

(Partnership OR "Joint-venture") AND ("Software outsourcing" OR "distributed software development") AND (factors OR characteristics OR promotes OR transfer) AND (vendors OR clients OR buyer)

\section{String2}

(Collaboration OR alliance) AND ("information system outsourcing" OR "IS-outsourcing") AND (drivers OR motivators OR leads OR convert OR Enter) AND ("Service-provider" OR "service-receiver" OR outsourcer)

\section{String3}

("Outsourcing partnership" OR GSD OR "Global software Development") AND ("information technology outsourcing" OR "IT-outsourcing") AND (elements OR parameters OR upgrade OR builds OR establish) AND (developer OR customer OR consumer)

\section{String4}

(Partnership OR "Joint-venture") AND ("Software outsourcing" OR "distributed software development") AND (risks OR barriers OR promotes OR transfer) AND (vendors OR clients OR buyer)

\section{String5}

(Collaboration OR alliance) AND ("information system outsourcing" OR "IS-outsourcing") AND (challenges OR "Negative impacts" OR leads OR convert OR Enter) AND ("Service-provider" OR "service-receiver" OR outsourcer)

\section{String6}

("Outsourcing partnership" OR GSD OR "Global software Development") AND ("information technology outsourcing" OR "IT-outsourcing") AND (obstacles OR hurdles OR upgrade OR builds OR establish) AND (developer OR customer OR consumer)

\subsection{Search validation}

Initially few relevant papers were found through the following major search terms using Google Scholar (www.scholar.google.com) SpringerLink(www.springerlink.com) and ScienceDirect (www.sciencedirect.com) ("Software Outsourcing" OR "IT outsourcing" OR "IS outsourcing") AND (partnership OR Collaboration OR factors OR risks OR Vendor OR Client).

Prior to undertake the review, these relevant papers will be used for the validation of the search strings.

\subsection{Search documentation}

Search results will be documented properly, and the following data will be recorded:

- Name of the database

- Search strategy

- Search Phase 
- Date of search

- Years covered (ALL)

- No. of publications found

- No .of publication selected

- Initial selection decision

- Final selection decision

\subsection{Search result Management}

Primary source references will be stored electronically in a separate directory. The result of each search will be stored as html pages.

\section{PUBLICATION SELECTION}

This section contains the following subsections.

- Inclusion Criteria

- Exclusion Criteria

- Selecting Primary Sources

- Publication Quality Assessment

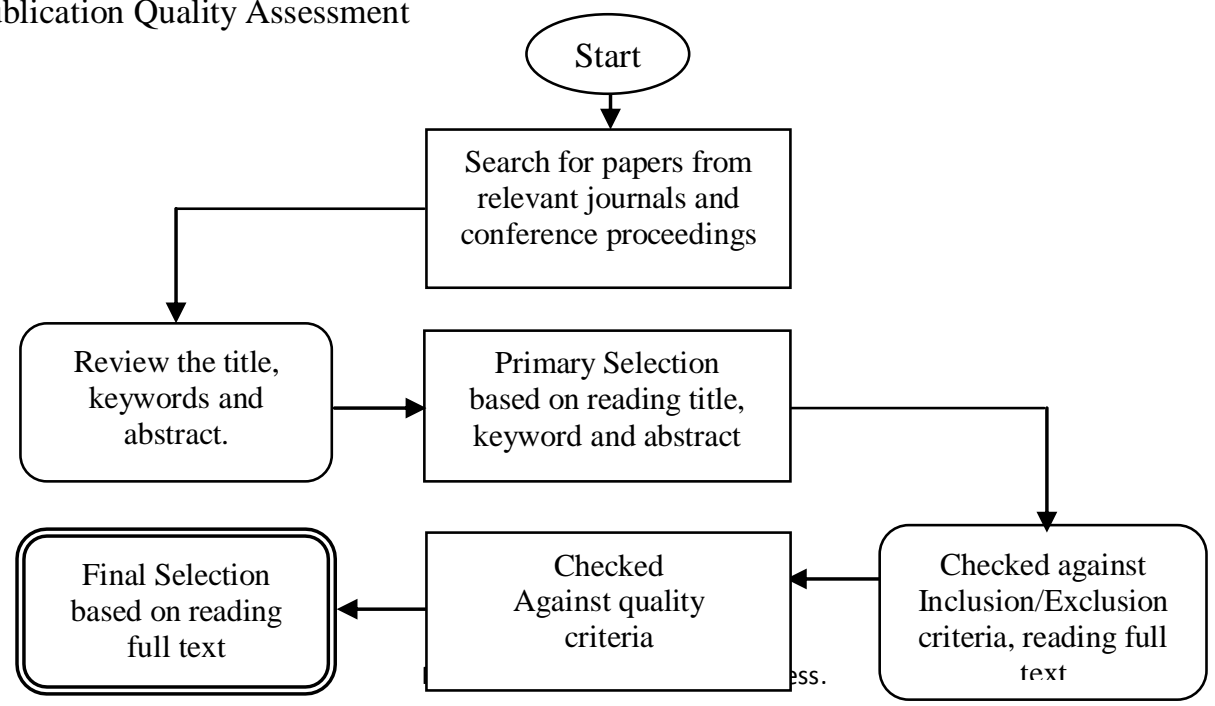

\subsection{Inclusion Criteria}

The selection of the literature (papers, technical reports, grey literature, etc), found through the search terms, will be performed using the following criteria:

- Studies that are reported in English language only.

- Studies that describe factors/motivators for software/IS/IT outsourcing partnership building.

- Studies that describe factors/motivators for software/IS/IT outsourcing relationship upgradation/promotion.

- Studies that describe criteria for a successful software outsourcing relationships.

- Studies that describes software/IS/IT outsourcing partnership.

- Studies that describes issues in software/IS/IT outsourcing relationship.

- Studies that describes issues in software/IS/IT outsourcing partnership.

- Studies that describe criteria for an outsourcing relationships upgradation/promotion/conversion.

- Studies that describe factors affecting the continuation/termination of the existing outsourcing relationship.

- Studies that evaluate vendor's capabilities for outsourcing partnership.

- Studies that describe risks in promoting the existing outsourcing relationship to partnership.

\subsection{Exclusion Criteria}

- Studies that are not relevant to the research questions.

- Studies that don't describe risks/problems in promoting to software outsourcing partnership.

- Studies that don't describe the factors that cause continuation/termination of the existing software outsourcing relationships.

- Studies that do not describe software outsourcing relationships.

- Studies other than outsourcing relationships. 


\subsection{Selecting Primary Sources}

The aim is to exclude only those results which have no relevance to the problem. Selection will be made as follow:

- Initial selection of primary sources will be performed by reviewing the title, keywords and abstract.

- This selection will be checked against the Inclusion/Exclusion criteria, by reviewing through full text.

- In case of any uncertainty, it will be forwarded to the secondary reviewer for guidance.

- The record of the Inclusion/Exclusion for each primary source will be maintained properly.

\subsection{Publication Quality Assessment}

The measurement of quality will be performed after final selection of publications. The quality checklist contains the following questions:

- Is it clear how the factors for establishing/building partnership between clients and vendors were identified?

- Is it clear how the factors for upgradation/promotion existing relationship between clients and vendors were identified?

- Is the author seems biased to publish positive results more than negative results?

- Is it clear how the risks in promoting existing outsourcing relationship to partnership were identified in outsourcing relationship?

- Has there sufficient data/sample size to support the findings?

Each of the above factors will be marked as 'YES', 'NO', 'Partially 'or N.A'. A secondary reviewer (my supervisor Dr Siffat Ullah Khan) will score a small subset for validation.

\section{DATA EXTRACTION STRATEGY}

The selected primary studies will be reviewed thoroughly in order to extract the data needed to answer the research questions [31].The following sections are considered in the data extraction process:

- Primary study data

- Data extraction process

- Data storage

\subsection{Primary Study Data}

The purpose of the study is to collect the data that address the research questions. The following data will be extracted from each publication.

- Publication details (Title, Authors, Reference)

- Data that address the research question

To address the research questions, the following data will be extracted.

RQ1: Background information, Critical success Factors (CSFs) for establishing/building software outsourcing partnership and Critical success Factors (CSFs) for promoting /upgrading existing outsourcing relationship into partnership.

RQ2: Background information, risks in promoting/upgrading existing outsourcing relationship into partnership. The below table show the types of data to be collected from each paper.

\subsection{Data Extraction Process}

The review will be undertaken by a single researcher, who will be responsible for the data extraction. A secondary reviewer will be consulted for guidance in case of an issue regarding the data extraction. Inter-rater reliability test will be performed for validation. 


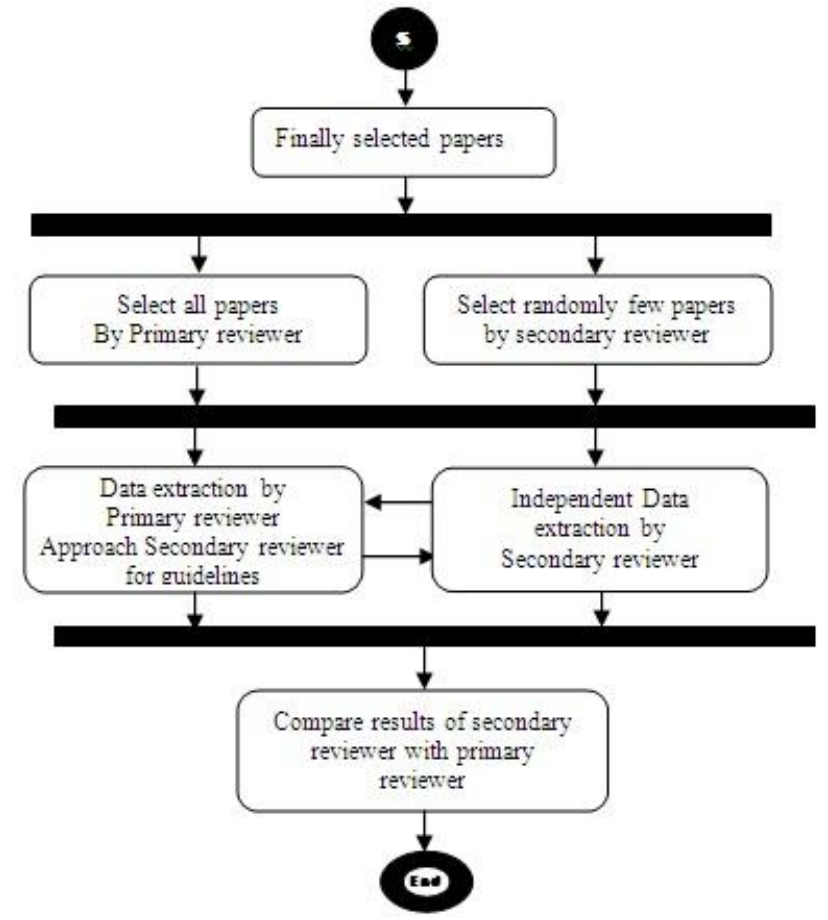

Fig. 3. Publication selection process.

\subsection{Data Storage}

The summarized data for each finally selected publication will be kept as a Microsoft Excel/SPSS document and will be stored electronically.

\section{VII.}

DATA SYNTHESIS

During the SLR, the extracted data will be synthesized in a way suitable for answering the questions.

\section{DATA TO BE EXTRACTED}

- Date of review

- Publication details (Title, Authors, Reference)

- Database

- Methodology (interview, case study, report, survey etc)

- Sample Population

- Target Population

- Publication Quality Description

- Organization Type (software house, university, research institute etc)

- Company size (small, medium, large)

- SPI Certification

- Country / location of the Analysis

- Year

- Critical Success Factors (CSFs) for establishing/building partnership: Factors that play an important role in building partnership between clients and vendors in software outsourcing relationship.

- Critical Success Factors (CSFs) for upgrading existing relationship into partnership: Factors that play an important role in upgrading existing relationship between clients and vendors in software outsourcing to partnership. 
Systematic Literature Review Protocol for Software Outsourcing Partnership (SOP)

For the reported SLR, due to two research questions, the synthesis will also be divided into two parts. For the Research Question1, the data will be synthesized by creating tables such that any background information is placed in one table, whereas the other table will include the data classifying CSFs having the columns (S.No, CSF's, Frequency, Percentages) showing the list of all the CSFs along with their frequencies and percentages.. For the Research Question2, the data will be synthesized by creating a table such that, any background information is placed in one table, whereas the other table will include the Risks having the columns (S.No, Risk, Frequency, Percentages) showing the list of all the Risks along with their frequencies and percentages

\section{VALIDATION OF THE PROTOCOL}

A preliminary draft protocol was initially submitted for review to my supervisor Dr Siffat Ullah Khan, who suggested some revisions. It was further reviewed by our fellows Asad Ullah Alam and Muhammad Ilyas Azeem. Finally the protocol was presented to the Software Engineering Research Group (www.SERG_UOM@yahoogroups.com) at University of Malakand Pakistan.

\section{REVIEW TIMETABLE}

\begin{tabular}{|c|c|}
\hline Task & Date \\
\hline Protocol Development & \\
\hline Start of the Protocol & 03-Oct-2011 \\
\hline Submission of the Protocol for Review & 15-Dec-2011 \\
\hline To address changes given by Supervisor/Re-submission & 10-Jan-2012 \\
\hline Completion of the Protocol & 31-Jan-2012 \\
\hline Protocol Validation & \\
\hline Presentation to SERG & 29-Feb-2012 \\
\hline To address changes given by SERG & 15-Mar-2012 \\
\hline External Reviewer & 01-May-2012 \\
\hline To address changes given by External Reviewer/ Re-submission & 15-May-2012 \\
\hline Implementation & \\
\hline Completion of Search & 03-July-2012 \\
\hline Completion of Primary Study Selection & 20-July-2012 \\
\hline Completion of Data Extraction & 30-Aug-2012 \\
\hline Completion of Data Synthesis & 20-Sep-2012 \\
\hline Completion of Review Report & $22-$-Oct-2012 \\
\hline
\end{tabular}

\section{CONCLUSION}

This paper presents the SLR protocol for the identification of factors for building or maintaining software outsourcing partnership between client and vendor organizations. The ultimate goal of this research is the development of software outsourcing partnership model (SOPM). The SLR is the first phase in the development of the stated model.

\section{DIVERGENCES}

In case of any divergence from the protocol, which may occur during the study, we will record any change in a new Appendix to this document.

\section{Acknowledgements}

We are thankful to software engineering research group (SERG_UOM), the reviewers and my fellows Abdul Wahid Khan, Asad Ullah Alam and Muhammad Ilyas Azeem for their assistance in the review process. 


\section{REFERENCES}

[1] J. N. Lee, Huynh, M.Q., Kwok, C.W., and Pi S.M., "The evolution of outsourcing research: What is the next issue?," presented at Proceeding of the Thirty-third Hawaii International Conference on Systems Sciences, Maui in Hawaii, 2000.

[2] W. F. a. N. McFarlan, R.L., "How to Manage an IT Outsourcing Alliance.," Sloan Management Review, vol. 36(2), pp. 9-23, 1995.

[3] M. A. Zviran, Niv Armoni,Aviad, "Building outsourcing relationship across the global community: the UPS-Motorola experience," Strategic information System, vol. 10, pp. 313-333, 2001.

[4] R. M. L.M. Applegate, E., "Kodak, Managing information systems through strategic alliances,," Harvard Business School Press, Boston, Boston 9-192-030,, 1991.

[5] B. F. Yang, Hongjiao Zuo,Meiyun, "A Case Study of Disaster Backup Outsourcing of SDB and Hi Sun," ICEC, Xi'an,China August 152005 .

[6] B. I. D.R. Lasher, S.L. Jarvenpaa,, "USAA-IBM partnerships in information technology: managing the image project," MIS Quarterly, vol. 15, pp. 551-565., 1991.

[7] K. D. L.M. Applegate, "Xerox: outsourcing global information technology resources," Harvard Business School Press, Boston 9195-158, 1995

[8] S. U Khan, M Niazi Ahmad,Rashid, "Factors influencing clients in the selection of offshore software outsourcing vendors," Journal of System and Software, 2010.

[9] J. T. V.Grover, M.Cheon, "The effect of service quality and partnership on the outsourcing of information system," Journal of Management information system, 1996.

[10] D. M. Lambert, M.A. Emmelhainz, and J.T. Gardner,, "Building Successful Logistics Partnerships," Journal of Business Logistics, vol. 20, pp. 165-181, 1999.

[11] J. N. a. K. Lee, Y. G,, "Effect of Partnership Quality on IS Outsourcing Success: Conceptual Frame work and Empirical Validation," Journal of Management Information Systems, vol. 15, pp. 29-61, 1999.

[12] R. Kishore, Rao, H.R., Nam, K., Rajagopalan, S., and Chaudhury, A., "A relationship perspective on IT outsourcing," Communications of the ACM, vol. 46(12), pp. 87-92, 2003.

[13] V. a. F. Michell, G., "The IT outsourcing market-place: vendors and their selection," Journal of Information Technology, vol. 12, pp. 223-237, 1997.

[14] R. a. B. Srinivasan, T.H., "Supplier Performance in Vertical Alliances: The Effects of Self-Enforcing Agreements and Enforceable Contracts," Organization Science, , vol. 17(4), pp. 436-452, 2006.

[15] B. F. Yang, Hongjiao Zuo,Meiyun, "The Integration Mechanism of IT Outsourcing Partnership," 2005.

[16] S. C. L. Yuan Sun, Tung Chen Sun,Pei, "The Factors Influencing Information Systems Outsourcing Partnership - A Study Integrating Case Study and Survey Research methods," presented at 35th Hawaii International Conference on System Sciences, 2002.

[17] D. P. Assmann, Teade, "Toward partnership in software subcontracting," computer in industry, vol. 54, pp. 137-150, 2003.

[18] J. K. Goo, Nam Rao,H. Raghav Song,Yongil, "An investigation of factors that influence the duration of IT outsourcing relationships," Decision Support Systems, pp. 19, 2006.

[19] T. Goles, \& Chin, W. W., "Information systems outsourcing relationship factors: detailed conceptualization and initial evdence," The DATA BASE for Advances in Information Systems, vol. 36, pp. 47-67, 2005.

[20] H. H. Olsson, Conchuir, E. O., Agerfalk, P. J., \& Fitzgerald, B., "Two-stage offshoring: an investigation of the Irish bridge," MIS Quarterly, vol. 32(2), pp. 257-279, 2008

[21] R. Klepper, \& Jones, W. O., "Outsourcing information technology." Systems and Services:, 1998.

[22] J.-N. Lee, Huynh, M. Q., \& Hirschheim, R., "An integrative model of trust on IT outsourcing: examining a bilateral perspective." Inf Syst Front, , vol. 10, pp. 145-163., 2008.

[23] T. W. Kern, L., "Exploring information technology outsourcing relationship: theory and practice," Strategic information System, pp. 321-350, 2000.

[24] L. W. G. Fitzgerald, "Contract and partnerships in the outsourcing of IT," presented at Proceedings of the 15th International Conference on Information Systems, British Colombia, Canada, 1994.

[25] J.C. Henderson, "Plugging into strategic partnerships: the critical IS connection,," Sloan Management Review, vol. 30(3), pp. 7-18, 1990 .

[26] J.-N. Lee, "The impact of knowledge sharing, organizational capability and partnership quality on IS outsourcing success," Information \& Management, pp. 323-335, 2001.

[27] D. E. R. Beulen, dr. Pieter, "Managing an IT-Outsourcing Partnership in Asia," presented at 35th Hawaii International Conference on System Sciences, 2002.

[28] C. S. Okoli, Kira, "A Guide to Conducting a Systematic Literature Review of Information Systems Research," Concordia University, Canada, Canada 1535-6078, 2010.

[29] B. C. Kitchenham, Stuart, "Guidelines for performing Systematic Literature Review in Software Engineering," Keele University, U.K 01, 9 July 20072007.

[30] M. C. Sarmad Ali Muhammad Ali Babar, Lianping Stol,Klaas-Jan, "A systematic review of comparative evidence of aspectoriented programming," Information and software Technology, vol. 52, pp. 871-887, 2010.

[31] M. Niazi, Staples, "Experiences using systematic review guidelines," The Journal of Systems and Software, vol. 80, pp. 1425-1437, 2006. 